\title{
Diagnosis of Pneumocystis carinii pneumonia in HIV antibody positive patients by simple outpatient assessments
}

\author{
Don E Smith, Alastair Forbes, Sandra Davies, Simon E Barton, Brian G Gazzard
}

\begin{abstract}
Background As increasing numbers of patients with immunosuppression induced by the human immunodeficiency virus (HIV) present with respiratory symptoms it is important to differentiate Pneumocystis carinii pneumonia from other chest diseases rapidly and start treatment early. The management of pneumocystis pneumonia could be improved if clinicians could diagnose this condition confidently on the basis of simple clinical assessments.

Methods Three hundred and eighteen patients with evidence of immunosuppression due to HIV infection and suspected pneumocystis pneumonia were investigated. A clinical history was taken and arterial blood gas analysis, chest radiography, oximetry during exercise, and sputum induction or bronchoscopy (or both) were performed.
\end{abstract}

Results Pneumocystis pneumonia was confirmed microscopically from induced sputum or bronchoalveolar lavage fluid in 154 patients; 118 had other chest disease. The remaining 46 patients had no definitive diagnosis. The best single independent predictors of a diagnosis of pneumocystis pneumonia were exercise induced oxygen desaturation and obvious interstitial infiltrates on the chest radiograph (odds ratios of 4.88 and 5.44 respectively). The symptom triad of exertional dyspnoea, cough, and fevers; the absence of pneumocystis pneumonia prophylaxis; and resting arterial hypoxaemia were less predictive (odds ratio $2.07,3.72$, and 0.69). An algorithm was developed that gave a positive predictive value for confirmed pneumocystis pneumonia of $95 \%$ and also identified those patients with a very small chance of having pneumocystis pneumonia (negative predictive value $85 \%$ ).

Conclusions The diagnosis of an initial episode of pneumocystis pneumonia can be confidently made in a large proportion of immunosuppressed patients with respiratory symptoms on the basis of clinical symptoms, the absence of prophylaxis, chest radiographic appearances, and oxygen desaturation during exercise as shown by oximetry. Using these simple features clinicians can rapidly assign patients to the appropriate type of management at presentation.

(Thorax 1992;47:1005-1009)

Pneumocystis carinii pneumonia remains the most common opportunist infection seen in patients with the acquired immunodeficiency syndrome (AIDS), ${ }^{1}$ occurring mainly in patients with strong laboratory and clinical evidence of immunosuppression. ${ }^{23} \mathrm{~A}$ definitive diagnosis of pneumocystis pneumonia can be made only by identifying this organism in induced sputum samples, ${ }^{4}$ which is time consuming, or in specimens obtained at bronchoscopy, ${ }^{5}$ which has known risks. Some non-invasive screening tests, including lung function testing, ${ }^{6}$ technetium-99m DTPA scanning, ${ }^{7}$ and exercise oximetry, ${ }^{8}$ have been developed to reduce the need for these investigations.

We have investigated whether simple information and investigations, which could be available at presentation, might obviate the need for definitive tests in a large proportion of patients by defining those in whom the diagnosis is extremely unlikely and those with such a high chance of having pneumocystis pneumonia that further investigation would be superfluous.

\section{Methods}

INVESTIGATIONS

A prospective assessment of all patients presenting to this hospital with suspected pneumocystis pneumonia was conducted. Patients were assessed either at a specialised HIV outpatient centre (Kobler Centre, St Stephen's Clinic) or during admission through the accident and emergency department. All patients with respiratory symptoms were evaluated if they had clinical markers of immunosuppression (oral candidiasis, oral hairy leucoplakia, or Kaposi's sarcoma), were known to have a recent CD4 ( $\mathrm{T}$ helper lymphocyte cell) count below $0.2 \times 10^{9} / 1,{ }^{3}$ or any previous HIV related illness. We included both those known to be HIV antibody positive and those presenting for the first time and suspected to be positive on the basis of clinical signs and symptoms of immunosuppression (if it was not known whether a patient had HIV antibodies and he subsequently declined an HIV antibody test or had a negative result he was excluded).

Information on exertional dyspnoea, cough, 
and fevers over the previous two weeks and on whether a recognised form of pneumocystis pneumonia prophylaxis had been taken over the preceding three months (nebulised pentamidine, oral co-trimoxazole, dapsone, or Fansidar (pyrimethamine-sulfadoxine)) was recorded on a case record form at the time the patient presented by either a doctor (DS) or a research nurse (SD). Patients then had chest radiography, arterial blood gas sampling, and exercise oximetry ${ }^{8}$ (performed immediately after chest radiography in patients seen in the outpatient clinic and within $\mathbf{4 8}$ hours in those patients admitted through the accident and emergency department).

Sputum induction and bronchoscopy (when sputum induction gave a negative result) were performed over the next five days by standard techniques. All the samples obtained were stained by Giemsa and silver (Grocott) and by immunofluorescent antibody stains ${ }^{9}$ (from mid 1988 onwards).

Routine sputum, induced sputum, and bronchoalveolar lavage samples were cultured for bacteria, fungi, and mycobacteria by standard methods.

\section{DEFINITIONS}

The following were defined as positive results for the purpose of analysis:

\section{Predictive clinical criteria}

1 A history of increasing dyspnoea, cough (dry or productive), and fevers within the preceding two weeks.

2 The absence of any pneumocystis pneumonia prophylaxis within the preceding three months.

3 An arterial oxygen tension of less than $11.0 \mathrm{kPa}$ while the patient was breathing room air (normal range $11 \cdot 7-15 \cdot 3 \mathrm{kPa}$ ).

4 A chest radiographic appearance of obvious diffuse perihilar or interstitial infiltrates. Equivocal changes, focal abnormalities, and normal chest radiographs were defined as negative.

5 A fall in arterial oxygen saturation $\left(\mathrm{SaO}_{2}\right)$ to $90 \%$ or below during standard testing. $\mathrm{SaO}_{2}$ was measured by an Ohmeda BIOX 3700 pulse oximeter during 10 minutes of exercise on an exercise cycle set to a standard resistance of $2 \mathrm{~kJ}$ (150 watts) and a standard speed of 20 $\mathrm{km} / \mathrm{h}$ (70 rev $/ \mathrm{min}){ }^{8}$ Patients unable to complete the full 10 minutes' exercise through coughing or muscular weakness whose $\mathrm{SaO}_{2}$ did not fall to $90 \%$ were analysed as though they had a negative result.

\section{Final diagnosis}

Confirmed pneumocystis pneumonia was defined as the presence of more than five typical cysts of $P$ carinii in an induced sputum or bronchoalveolar lavage sample.

"Clinically suspected pneumocystis pneumonia" was defined on the basis of suggestive symptoms that responded to standard antipneumocystis pneumonia treatment when no other diagnosis was uncovered but $P$ carinii organisms were not found in induced sputum or bronchoalveolar lavage fluid.

Pneumocystis pneumonia was considered to be excluded in those patients with a negative induced sputum or bronchoscopy sample who did not require pneumocystis pneumonia treatment within the following three months, and in those found to have other disease and no evidence of pneumocystis pneumonia. Pulmonary Kaposi's sarcoma was diagnosed on the basis of typical bronchoscopic appearances.

\section{STATISTICAL ANALYSIS}

The $\chi^{2}$ test with Yates's correction was used to compare differences in proportions for categorical data. Differences in continuous data were evaluated by Student's $t$ test (EPI-Info 5.0 software package $\left.{ }^{10}\right)$. Odds ratios were used to estimate the risk of pneumocystis pneumonia associated with the presence of each variable. Multivariate logistic regression analysis was used to identify the independent predictive risk of pneumocystis pneumonia for each variable (Statistical Analysis Software). Sensitivities, specificities, and positive predictive values were calculated for each variable and for combinations of variables.

\section{Results}

Of the 318 evaluated patients, 310 were homosexual or bisexual men $(97 \%)$, four were heterosexual women, and four were heterosexual male intravenous drug users. Pneumocystis pneumonia was confirmed in 154 patients and "clinically suspected" in a further 46. These latter patients were excluded from further analysis as no definitive diagnosis could be made, but including them as having confirmed pneumocystis pneumonia would not have altered any of the statistical associations presented here. Other diseases were diagnosed in 118 patients (table 1 ).

There were no significant differences in age or median CD4 counts at the time of assessment between patients with pneumocystis pneumonia ( 35.8 years, and $50 \times 10^{4}$ cells $\left./ 1\right)$ and those with other chest diseases $(35.6$ years and $4.6 \times 10^{4}$ cells/1). A history of previous pneumocystis pneumonia was obtained in $16 \%$ of patients with pneumocystis pneumonia as their current illness and $22 \%$ of patients with

Table 1 Final diagnoses in 318 patients presenting with suspected pneumocystis pneumonia

\begin{tabular}{lc}
\hline & $\begin{array}{l}\text { No of } \\
\text { patients }\end{array}$ \\
\hline Confirmed pneumocystis pneumonia & 154 \\
Clinically suspected pneumocystis pneumonia & 46 \\
\cline { 2 - 2 } & 200 \\
Bacterial chest function & 68 \\
Pulmonary Kaposi's sarcoma & 14 \\
Mycobacterium avium-intracellulare & 10 \\
Bronchial candidiasis & 7 \\
Pulmonary tuberculosis & 5 \\
Lymphoma & 3 \\
Symptomatic anaemia & 3 \\
Septicaemia & 2 \\
Asthma & 2 \\
Histoplasmosis & 1 \\
Cytomegalovirus pneumonitis & 1 \\
Disseminated blastomycosis & 1 \\
Myopathy & 1 \\
& 118 \\
Total & 318 \\
\hline
\end{tabular}


Table 2 Univariate and multivariate risk of pneumocystis pneumonia in two hundred and seventy two HIV infected patients with respiratory symptoms

\begin{tabular}{lll}
\hline Test & $\begin{array}{l}\text { Univariate } \\
\text { odds ratio }(95 \% \text { CI })\end{array}$ & $\begin{array}{l}\text { Multivariate } \\
\text { odds ratio }(95 \% \text { CI }\end{array}$ \\
\hline Classical symptoms & $2 \cdot 79(1 \cdot 61-4 \cdot 83)^{\star \star}$ & $2 \cdot 07(1 \cdot 01-4 \cdot 25)^{\star}$ \\
No pneumocystis pneumonia prophylaxis & $3 \cdot 67(2 \cdot 10-6 \cdot 43)^{\star \star \star}$ & $3 \cdot 72(1 \cdot 8-7 \cdot 7)^{\star \star}$ \\
Arterial hypoxaemia & $1 \cdot 64(0 \cdot 96-2 \cdot 83)$ & $0 \cdot 69(0 \cdot 33-1 \cdot 44)$ \\
Interstitial shadowing & $7 \cdot 13(3 \cdot 91-13 \cdot 0)^{\star \star \star}$ & $4 \cdot 89(2 \cdot 18-10 \cdot 91)^{\star \star \star}$ \\
Desaturation & $7 \cdot 85(4 \cdot 41-13 \cdot 99)^{\star \star \star}$ & $5 \cdot 44(2 \cdot 62-11 \cdot 25)^{\star \star \star}$ \\
\hline
\end{tabular}

${ }^{\star} \mathrm{p}<0.05 ;{ }^{\star \star} \mathrm{p}<0.001 ;{ }^{\star \star \star} \mathrm{p}<0.0001$

other chest diseases. Fewer patients with than without pneumocystis pneumonia were smokers $(45 \% v 57 \%)$, though the difference was not significant.

Univariate analysis showed that chest radiographic features, desaturation during exercise, absence of prophylaxis $(p<0.001)$ and classicial clinical symptoms $(p<0.001)$ were strongly predictive of pneumocystis pneumonia. Despite obvious potential associations between these features, multivariate analysis also showed that they remained strongly predictive as independent variables (table 2). Arterial hypoxaemia, however, did not differentiate pneumocystis pneumonia from other chest diseases $(p=0.07)$ and had a low predictive value (fig 1 ). The predictive value of these features did not differ between smokers and non-smokers.

Exercise induced oxygen desaturation was the single most useful parameter (fig 1), with a multivariate odds ratio for confirmed pneumocystis pneumonia of $5 \cdot 43$. Although patients were asked to continue exercising for a full 10 minutes, only $38 \%$ of patients with pneumocystis pneumonia were able to achieve this compared with $61 \%$ of patients without pneumocystis pneumonia. In $80 \%$ of patients with pneumocystis pneumonia $\mathrm{SaO}_{2}$ fell to $90 \%$ or less within the first three minutes of the test. Only 13 patients with pneumocystis pneumonia completed a full 10 minute test without developing desaturation.

Although diffuse interstitial shadowing was sensitive, it was not specific for pneumocystis pneumonia as similar infiltrations were seen in $60 \%$ of patients with pulmonary tuberculosis and $39 \%$ of patients with pulmonary Kaposi's sarcoma. Only $11 \%$ of patients with atypical mycobacteriosis, $7 \%$ of those with bronchial

Table 3 Effect of a prior episode of pneumocystis pneumonia (PCP)

\begin{tabular}{lcc}
\hline & \multicolumn{2}{l}{ Current diagnosis $($ No $(\%))$} \\
\cline { 2 - 3 } & $P C P$ & Non-PCP \\
\hline No previous pneumocystis pneumonia (n) & 133 & 92 \\
Classical symptoms & $102(77)$ & $40(43)^{\star}$ \\
Having prophylaxis & $25(19)$ & $69(75)^{\star}$ \\
Hypoxaemic & $77(58)$ & $42(46)$ \\
Obvious chest infiltrates & $84(63)$ & $12(13)^{\star}$ \\
Desaturation & $109(82)$ & $16(17)^{\star}$ \\
Previous pneumocystis pneumonia (n) & 24 & 26 \\
Classical symptoms & $12(50)$ & $18(68)$ \\
Having prophylaxis & $18(75)$ & $23(88)$ \\
Hypoxaemic & $11(46)$ & $9(35)$ \\
Obvious chest infiltrates & $8(33)$ & $7(25)$ \\
Desaturation & $18(75)$ & $20(75)$ \\
Total & 154 & 118
\end{tabular}

${ }^{\star} \mathrm{p}<0.00001$ by $\chi^{2}$ test with Yates's correction.
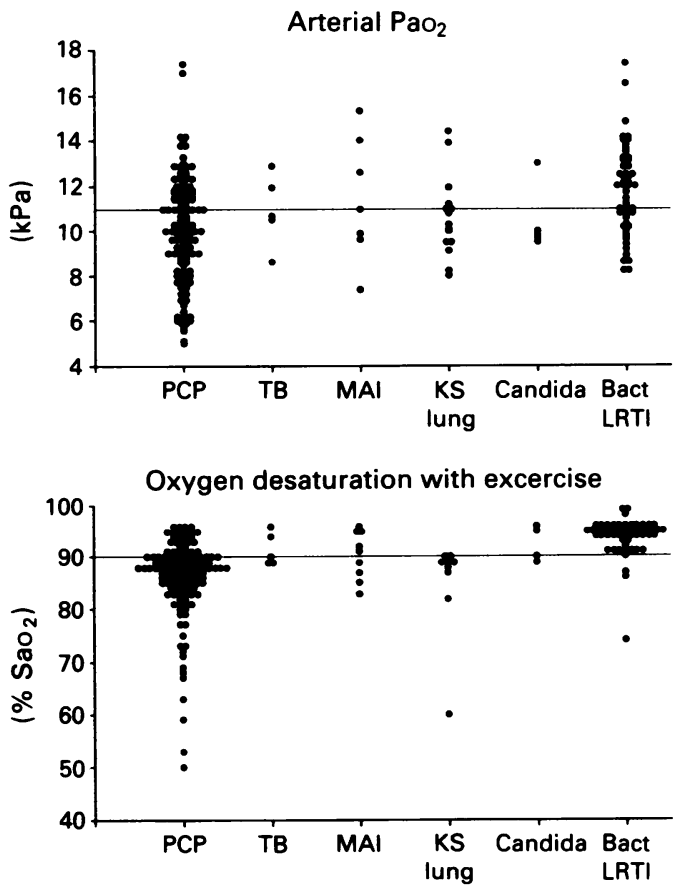

Figure 1 -Individual resting arterial oxygen pressures ( $\mathrm{kPa}$ ) for patients with pneumocystis pneumonia (PCP), pulmonary tuberculosis (TB), Mycobacterium avium-intracellulare (MAI), pulmonary Kaposi's sarcoma (KS), bronchial candidiasis (Candida), or bacterial lower respiratory tract infections (Bact LRTI). The few points above the normal range are for patients breathing room air but may represent sampling or technical errors. B-Individual minimum oxygen saturation $\left(\mathrm{SaO}_{2}\right)$ percentages during exercise for patients with Pneumocystis pneumonia (PCP), pulmonary tuberculosis (TB), Mycobacterium avium-intracellulare (MAI), pulmonary Kaposi's sarcoma (KS), bronchial candidiasis (Candida), or bacterial lower respiratory tract infections (Bact LRTI).

candidiasis, and $2 \%$ of those with bacterial chest infections had similar infiltrates.

None of the features studied was useful in predicting pneumocystis pneumonia in patients who had had at least one previous confirmed episode of pneumocystis pneumonia (table 3).

With the exception of hypoxaemia, the positive predictive value for a correct diagnosis of pneumocystis pneumonia was improved by combining two or more of the defined criteria (table 4). The triad of typical symptoms, arterial hypoxaemia, and diffuse chest infiltrates together gave a high positive predictive value $(88 \%)$, but were present in only one third of the patients with confirmed pneumocystis pneumonia.

An algorithm of management was developed from the positive predictive values for combinations of features (fig 2). Typical symptoms and knowledge of pneumocystis pneumonia prophylaxis were combined as they will almost always be available from a clinical history. This algorithm was used to place patients into three categories: (1) pneumocystis pneumonia highly likely; (2) chest disease uncertain; (3) pneumocystis pneumonia highly unlikely. For these categories three corresponding management strategies were proposed-namely, start pneumocystis pneumonia treatment, investigate further, treat for bacterial infection, and observe. Had this algorithm been used, empirical treatment would have been correctly 
Table 4 Sensitivity (Sens), specificity (Spec), and positive predictive values (PPV) in 272 patients for individual and combined predictors of pneumocystis pneumonia

\begin{tabular}{lccc}
\hline Individual features & $\begin{array}{l}\text { Sens } \\
(\%)\end{array}$ & $\begin{array}{l}\text { Spec } \\
(\%)\end{array}$ & $\begin{array}{r}P P V \\
(\%)\end{array}$ \\
\hline Classical symptoms & 73 & 51 & 167 \\
No pneumocystis pneumonia prophylaxis & 72 & 58 & 70 \\
Arterial hypoxia & 56 & 56 & 66 \\
Interstitial shadowing & 58 & 84 & 83 \\
Desaturation & 74 & 73 & 77
\end{tabular}

Combination of features

Symptoms + no prophylaxis

Symptoms + hypoxaemia

Symptoms + desaturation

Symptoms + DISS

No prophylaxis + hypoxaemia

No prophylaxis + desaturation

No prophylaxis + DISS

Hypoxaemia + desaturation

Hypoxaemia + DISS

Desaturation + DISS

Symptoms + no prophylaxis + hypoxaemia

Symptoms + no prophylaxis + desaturation

Symptoms + no prophylaxis + DISS

No prophylaxis + hypoxaemia + desaturation

Symptoms + hypoxaemia + DISS

Symptoms + no prophylaxis + hypoxaemia + DISS

Symptoms + no prophylaxis + desaturation + DISS

All five criteria met

\begin{tabular}{rrr}
55 & 81 & 80 \\
43 & 75 & 74 \\
59 & 82 & 81 \\
46 & 91 & 88 \\
45 & 79 & 78 \\
62 & 91 & 91 \\
46 & 97 & 94 \\
49 & 83 & 81 \\
43 & 89 & 85 \\
49 & 91 & 91 \\
36 & 89 & 84 \\
46 & 97 & 95 \\
38 & 98 & 96 \\
31 & 97 & 94 \\
35 & 93 & 88 \\
29 & 98 & 98 \\
25 & 100 & 100 \\
21 & 100 & 100 \\
\hline
\end{tabular}

Symptoms-exertional dyspnoea plus cough plus fevers; no prophylaxis-not taken a recognised form of pneumocystis pneumonia prophylaxis within the previous three months; hypoxia-arterial oxygen tension below $11.0 \mathrm{kPa}$; desaturation-drop in oxygen saturation to below $90 \%$ with exercise; DISS-obvious diffuse interstitial infiltrates on the chest radiograph.

instigated in 73 patients with pneumocystis pneumonia (95\% accuracy); four patients with other diseases would have been incorrectly given pneumocystis pneumonia treatment (two with tuberculosis and two with bacterial chest infections). Forty seven patients would have been assigned to the " unlikely" category; of these, seven in fact had pneumocystis pneumonia ( $85 \%$ accuracy). Forty eight per cent of those patients requiring further investigations had an eventual diagnosis of pneumocystis pneumonia.

\section{Discussion}

Our intention in this study was to assess the usefulness of readily available data in making a diagnosis of pneumocystis pneumonia in patients infected with HIV before embarking

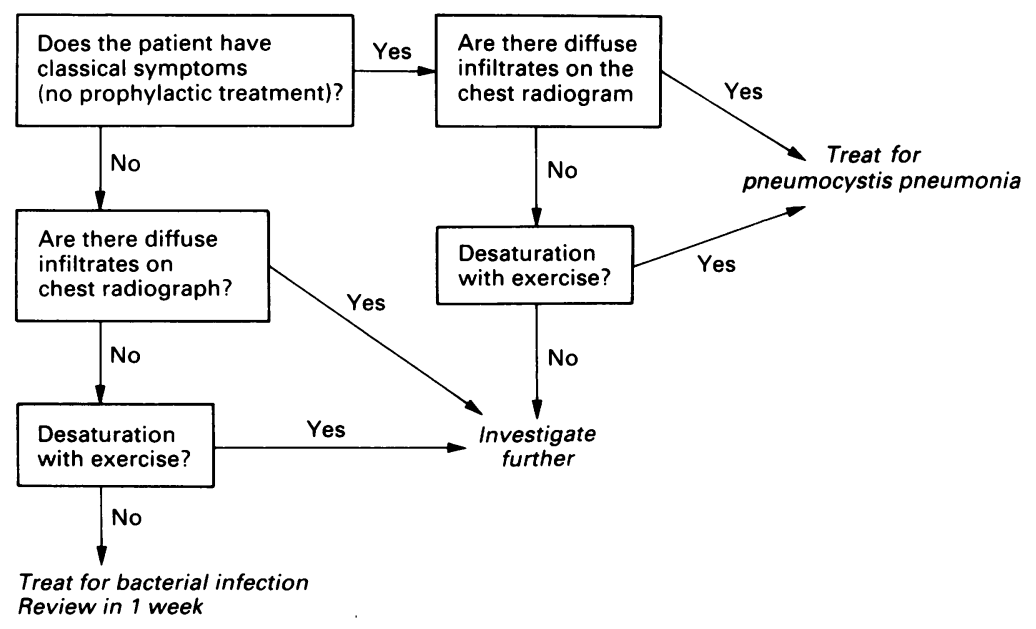

Figure 2 Proposed algorithm for the diagnosis of pneumocystis pneumonia in immunosuppressed patients infected with HIV. on more extensive or invasive investigations. As pneumocystis pneumonia is most unlikely in the early stages of HIV infection, the sensitivity and specificity of simple features are likely to be of little clinical relevance in this group. Only patients with signs of immunosuppression or a known reduced $\mathrm{CD} 4$ count were therefore studied. We hoped that this would provide useful predictive information in a group of patients with advanced immunosuppression in whom the prior probability of pneumocystis pneumonia was relatively high. ${ }^{2}$

The five pieces of information were individually of limited predictive value in the accurate diagnosis of pneumocystis pneumonia. Although the absence of pneumocystis pneumonia prophylaxis and the presence of interstitial shadowing on the chest radiograph were sensitive tests, both were relatively non-specific. Arterial hypoxaemia does not provide a sensitive test as it is present in various other respiratory conditions; but arterial blood gas analyses are still needed during the initial evaluation to deterine whether adjunctive corticosteroid treatment is necessary. ${ }^{11}$

Exercise desaturation was the most sensitive single index of pneumocystis pneumonia, but was less specific than an indicative chest radiograph, largely because desaturation recurred in two thirds of patients who had had pneumocystis pneumonia previously, whatever the cause of the current respiratory problem. Exercise oximetry contributes most in those patients who have not had previous pneumocystis pneumonia and those without obvious radiographic changes.

None of the features studied was useful in prediciting pneumocystis pneumonia in patients who had at least one previously confirmed episode. Although the increased use of prophylaxis in this subgroup is easily explained, the exercise desaturation patterns are more difficult to interpret. The most likely reason for this is that inflammatory scarring and thickening of the alveolar interstitium occurs during resolution of the initial episode of pneumocystis pneumonia, resulting in some degree of respiratory impairment whenever a subsequent pulmonary insult ensues.

The proposed algorithm was devised on the pragmatic basis that the chest radiograph, the arterial blood gas tensions, and the history at presentation will almost always be immediately available to the clinician. This combination provides highly specific features but with a relatively low sensitivity. The specificity is further increased if results of exercise desaturation are also positive, and the criterion of replacing low resting arterial blood gas oxygen tension with exercise induced oxygen desaturation greatly improves the overall diagnostic usefulness of these simple tests.

Further definitive tests for pneumocystis pneumonia may be considered unnecessary in patients assigned by the algorithm to the category "pneumocystis pneumonia highly likely" (positive predictive value over 95\%) and many clinicians would wish to treat such patients even if subsequent tests gave negative 
results. Similarly, the negative predictive value of $85 \%$ in the "pneumocystis pneumonia unlikely" category identifies a group of patients with such a low likelihood of pneumocystis pneumonia that it is justifiable to manage them by close observation or treating them for a presumed bacterial chest infection (or both). If used in this way the algorithm would have obviated the need for further investigations in $59 \%$ of all the patients seen.

Other screening tests, including DTPA scanning, ${ }^{7}$ gallium scanning, ${ }^{12}$ computed tomography of the chest, ${ }^{13}$ and determination of carbon monoxide transfer factor ${ }^{14}$ or serum lactate dehydrogenase activity, ${ }^{15}$ have been advocated to help in the diagnosis of pneumocystis pneumonia and therefore reduce the need for further investigations. Most such tests, which are either sensitive or specific, are expensive or unavailable outside specialist centres, or yield results only after some delay.

In conclusion, this algorithm has been developed to provide a simple and rapid assessment of the probability of pneumocystis pneumonia in patients with advanced HIV disease. Empirical treatment may be confidently instigated on the basis of risk features ascertained at the time the patient first presents, thereby enabling treatment to start earlier and survival, it is hoped, to improve. It should also reduce the number of patients requiring more extensive screening and reduce the requirement for invasive definitive tests.

We wish to thank Dr P Easterbrook (AIDS research unit, St Stephen's Clinic) and Dr K MacRae (department of professorial medicine, Charing Cross Hospital) for their statistical advice.

1 Selik RM, Starcher ET, Curran JW. Opportunistic diseases reported in AIDS patients: frequencies, associations, and trends. AIDS 1987;1:175-82.

2 Phair J, Munoz A, Detels R, Kaslow R, Rinaldo C, Saah A. The risk of Pneumocystis carinii pneumonia among men infected with human immunodeficiency Virus type 1.
Multicenter AIDS cohort study group. $N$ Engl $J$ Med 1990;322:161-5.

3 Masur H, Ognibene FP, Yarchaon R, Shelhamer JH, Baird $\mathrm{BF}$, Travis W, et al. CD4 counts as predictors of opportunistic pneumonias in human immunodeficiency virus (HIV) infection. Ann Intern Med 1989;111:223-31.

4 Bigby TD, Margolskee D, Curtis JL, Michael PF, Sheppard $\mathrm{D}$, Hadley WK, et al. The usefulness of induced sputum in the diagnosis of Pneumocystis carinii pneumonia in patients with the acquired immunodeficiency syndrome. Am Rev Respir Dis 1986;133:515-8.

5 Coleman DL, Dodek PM, Luce JM, Golden JA, Gold WM, Murray JF. Diagnostic utility of fiberoptic bronchoscopy in patients with Pneumocystis carinii pneumonia and the acquired immune deficiency syndrome. Am Rev Respir Dis 1983;128:795-9.

6 Stover DE, White DA, Romano PA, Gellene RA, Robeson WA. Spectrum of pulmonary diseases associated with the acquired immune deficiency syndrome. $A m J$ Med 1985;78:429-37.

7 Leach RM, Davidson AC, O'Doherty MJ, Nayagam M, Tang A, Bateman NT. Non-invasive management of fever and breathlessness in HIV positive patients. Eur Respir $J$ 1991;4:19-25.

8 Smith DE, McLuckie A, Wyatt J, Gazzard B. Severe exercise hypoxaemia with normal or near normal X-rays: a feature of Pneumocystis carinii infection. Lancet 1988;ii:1049-51.

9 Elvin KM, Bjorkman A, Linder E, Heurlin N, Hjerpe A. Pneumocystis carinii pneumonia: detection of parasites in sputum and bronchoalveolar lavage fluid by monoclonal antibodies. $B M J$ 1988;297:381-4.

10 Dean AD, Dean JA, Burton JH, Dicker RC. EPI Info, version 5: a word processing, database, and statistics program for epidemiology on micro-computers. Atlanta, Georgia: Centers for Disease Control, 1990.

11 Consensus statement on the use of corticosteroids as adjunctive therapy for pneumocystis pneumonia in the acquired immunodeficiency syndrome. The National Institutes of Health-University of California expert panel for corticosteroids as adjunctive therapy for Pneumocystis pneumonia. N Engl J Med 1990;232:1500-4.

12 Tuazon CU, Delaney MD, Simon GL, Witorsch P, Varma VM. Utility of Gallium ${ }^{67}$ scintigraphy and bronchial washings in the diagnosis and treatment of Pneumocystis carinii pneumonia in patients with the acquired immune deficiency syndrome. Am Rev Respir Dis 1985;132: 1087-92.

13 Hartelius H, Gaub J, Ingemann-Jensen L, Faber V. Computed tomography of the lungs in acquired immunodeficiency syndrome. An early indicator of interstitial pneumonia. Acta Radiol 1988;29:641-4.

14 Shaw RJ, Roussak C, Forster SM, Harries R, Pinching AJ Mitchell DM. Lung function abnormalities in patients infected with the human immunodeficiency virus with and without overt pneumonitis. Thorax 1988;43:436-40.

$15 \mathrm{Katz} \mathrm{MH}$, Baron RB, Grady D. Risk stratification of ambulatory patients suspected of pneumocystis pneumonia. Arch Intern Med 1991;151:105-10. 